\title{
Assessment and Modification of the Tinnitus-Related Cortical Network
}

\author{
Winfried Schlee, Dipl.-Psych., ${ }^{1}$ Katalin Dohrmann, Ph.D., 1 \\ Thomas Hartmann, Dipl.-Psych., ${ }^{1}$ Isabel Lorenz, Dipl.-Psych., ${ }^{1}$ \\ Nadia Müller, Dipl.-Psych., 1 Thomas Elbert, Ph.D.,' \\ and Nathan Weisz, Ph.D.2
}

\section{ABSTRACT}

Tinnitus refers to the perception of a sound in the absence of any physical source, and it is widely believed that this phantom sound is generated in the central nervous system. Thus the activation of neuronal. cell assemblies is chronically changed in patients with an ongoing tinnitus perception. We used magnetoencephalography to investigate these changes in a resting condition. There was an increase of synchronized activity in the gamma and delta frequency range together with a decrease in the $\alpha$ band. Manipulation of these cortical networks by means of neurofeedback therapy resulted in a reduction of tinnitus loudness and distress. In this article we review the basic research and the clinical studies conducted in our laboratory and propose a model that explains the results and helps guide future research and therapy.

KEYWORDS: Tinnitus, magnetoencephalography, neurofeedback, resting state

Learning Outcomes: As a result of this activity, the participant will be able to (1) describe pathological changes in the activity of neuronal cell assemblies in chronic tinnitus patients, and (2) to use this knowledge to conduct EEG neurofeedback for the treatment of chronic tinnitus.

Subjective tinnitus is described as a conscious perception of a sound in the absence of any physical sound source. This sound is typically described by the patients as a tone, a hissing or roaring noise, or in some cases as a combination of several sounds. Transient tinnitus is quite common in the general population and lasts typically a few seconds to a few hours or days. However, chronic tinnitus is diagnosed when the subject perceives the tinnitus for $>3$ months. About 5 to $15 \%$ of the population in Western societies ${ }^{1}$ report chronic tinnitus, and in 1 to $3 \%$ the tinnitus affects quality of life by disturbing sleep, impairing one's ability to
${ }^{1}$ Department of Psychology, University of Konstanz, Konstanz, Germany; ${ }^{2}$ Dynamique cérébrale et cognition, INSERM U 821, Lyon, France.

Address for correspondence and reprint requests: Winfried Schlee, Dipl.-Psych., Department of Psychology, University of Konstanz, PO Box D25, 78458 Konstanz, Germany (e-mail: winfried.schlee@uni-konstanz.de).
Tinnitus: Part I; Guest Editors, Richard Salvi, Ph.D., Wei Sun, Ph.D., and Edward Lobarinas, Ph.D. 
concentrate at work, and affecting social interactions, as well as causing psychiatric distress. ${ }^{2}$ Several programs are currently available that help chronic tinnitus patients cope with their tinnitus sensation, or adapt to it, but there is still no effective cure that eliminates the tinnitus.

Many affected individuals are able to localize their tinnitus to one or both ears, and in most cases the tinnitus sensation is accompanied by an audiometrically measurable damage to the cochlea. Thus one may think that the tinnitus is generated in the ears; however, this is most likely not the case. If the phantom sound was generated in the ears, a transection of the auditory nerve would reliably eliminate the ongoing perception of the tinnitus sound. To date, there is much evidence refuting this view. In only a small percentage of patients does the auditory nerve section lead to relief from tinnitus, and the majority of patients still experience tinnitus after the surgery. ${ }^{3,4}$ Furthermore, Berliner and colleagues ${ }^{5}$ report that in $50 \%$ of a sample of patients without tinnitus, the surgical removal of an acoustic tumor resulted in tinnitus after the operation. Also, if the tinnitus was generated in the periphery, a systematic enhancement of spontaneous activity in auditory nerve fibers would be present. As summarized by Eggermont and Roberts, ${ }^{6}$ changes of spontaneous firing rate in the auditory nerve is rather unsystematic. The technique used to induce tinnitus in animals affects the outcome with a decrease, an enhancement, or no change of spontaneous auditory nerve activity. Regardless of the technique used, all forms led to tinnitus in the animal. These results suggest that for the majority of cases the sensation of tinnitus originates in central rather than from peripheral parts of the auditory system. A large body of studies demonstrates the importance of central structures in tinnitus. Tinnitus-related changes of the spontaneous activity can be found throughout the central auditory system. The spontaneous firing rate is enhanced in the dorsal cochlear nucleus, the inferior colliculus, and the primary and the secondary auditory cortex. ${ }^{6}$ But altered activity also can be found in nonauditory structures. Mirz and colleagues reported a reduction of the regional cerebral blood flow when the tinnitus sensation was suppressed by lidocaine or masking. These sup- pressions were mainly located in the middle frontal, the middle temporal, and the posterior sites of the right hemisphere. ${ }^{8}$

In addition to alterations of cortical activity, there also are reports on structural changes of the central nervous system in tinnitus $\mathrm{pa}^{-}$ tients. In a voxel-based morphometry study, Mühlau showed a decrease of gray matter density in subcallosal regions and a gray matter increase in the posterior thalamus and the medial geniculate nucleus for tinnitus subjects compared with healthy controls. ${ }^{9}$ Investigating the structural connectivity of the temporal cortex with frontal and parietal sites via diffusion tensor imaging, Lee et al demonstrated a reduction of white matter density for the left frontal and right parietal arcuate fasciculus in tinnitus patients. ${ }^{10}$

The most influential hypothesis on how tinnitus is generated in the cortex is based on reorganizational processes within the auditory system. Animal and human research has shown that profound hearing loss can lead to a reorganization of the tonotopic organization in the auditory cortex. ${ }^{6,11,12}$ However, there is no clear relationship between hearing loss and the tinnitus sensation, given that a considerable number of patients with hearing loss do not suffer from tinnitus. König and colleagues ${ }^{13}$ analyzed the audiograms of two groups of patients that all suffered from noise-induced hearing loss: one group with and one group without tinnitus. In this sample, the tinnitus group showed even a smaller amount of hearing loss than the nontinnitus group. However, looking at the shape of the audiogram curve, they found the maximum steepness of the audiogram to be greater in patients with tinnitus than in patients without tinnitus. Conceptually, a steeper slope would translate into a more abrupt transition from normally afferented to deprived tonotopic regions. How this state enhances the likelihood of producing tinnitus-relevant neuronal activity is unknown. More research is needed to understand the relationship between hearing loss and cortical reorganization at the primary auditory cortex, which also includes knowledge about the contribution of damage to the inner and outer hair cells in the cochlea.

To understand further the cortical mechanisms that underlie tinnitus, we conducted 
several studies investigating the spontaneous oscillatory activity of the human cortex in subjects with and without an ongoing perception of tinnitus. Using magnetoencephalography (MEG) and electroencephalography (EEG), we measured electric activity in large populations of neurons. Simultaneous activation of a large number of neurons can be understood as a weighting mechanism of the brain whereby the more relevant information is conducted by cell assemblies that fire in synchrony. Due to technical constraints, described in more detail later, MEG and EEG record signals from the brain only if the neuronal population is large ( $>10,000$ neurons) and if they are activated in sync.

In the resting state, the most relevant difference between a group of healthy controls and tinnitus patients is the perception of a phantom sound in the latter group. Note that the term "spontaneous activity" in this context refers to synchronized activity of the human cortex while the subjects are resting without any cognitively demanding task. Whereas the "spontaneous activity" or "spontaneous firing rate" in animal research typically refers to spontaneous discharges of single or multiple neurons that may, or may not be, in sync. In the following we outline in brief the basic principles of recording cortical activity via EEG/ $M E G$ and provide a review on recent findings in our laboratory.

\section{CORTICAL ACTIVITY IN INEURAL NETWORKS}

The human brain consists of $\sim 10^{10}$ neurons with an estimated $10^{14}$ connections among them. Thus each neuron is connected to $\sim 10^{4}$ other neurons. The vast majority of these neurons are located in cortical layers and form a complex network of rich connectivity. Most of the input to cortical cells arrives from other cortical cells as described by Braitenberg and Schutz, ${ }^{14}$ who estimate that $99 \%$ of all connections are intracortical connections. As suggested by Hebb, ${ }^{15}$ these neurons are organized in neural cell assembles (NCAs) that typically connect neurons in the close neighborhood to each other but also make long-range connections to other NCAs. Thus the anatomical properties of brain networks share the characteristics of small-world networks. ${ }^{16,17}$ We can thus view the neurons as nodes, and the connections between them as links of the network. One of the most important features of smallworld networks is that information can travel within such a network very efficiently from one node to another. ${ }^{18}$

The principle of small-world networks has been found in anatomical as well as in functional descriptions of neural networks. ${ }^{16}$ The functional description of neural networks does not refer to physical connectivity; rather, it describes the relationship among the observed cortical activities. MEG and EEG only allow for assumptions of the functional properties of neural networks. In recent years, several studies have demonstrated small-world properties of functional neural networks using MEG and EEG. ${ }^{19-21}$ Within this framework, Bassett and colleagues $^{19}$ were able to show that the architecture of the functional neuronal network appears to be different for various frequency bands. To circumscribe these networks fully, we need to integrate information of their local and global properties specific to different frequency bands.

\section{MEASURING CORTICAL ACTIVITY}

The idea of measuring electrical activity of the human brain can be traced back to Hans Berger, ${ }^{22}$ who did the pioneering work in recording small electrical voltages between electrodes attached to the head of the subject. If a single electrode is placed at the scalp, it measures the spatial summation of electric potentials of the underlying tissue. The electric activity of a neuronal population generates a local field potential, which can, if great enough, be detected as a power change at the surface. Because the potential of a single active neuron is too weak to produce a signal in a superficial electrode, a summation of at least 10,000 neurons is needed. These electrical potentials must be modulated in synchrony, and all currents must point in the same direction to create a sufficiently strong local field potential. Thus power enhancements recorded in EEG/MEG reflect local synchronization of a large neuronal population. As a consequence, oscillating activity of a neuronal cell assembly with a high 
percentage of short-range connections in the close neighborhood should lead to an increase of local synchrony measures (i.e., amplitude enhancement in the frequency of the oscillation) on the surface.

To investigate long-range connections of NCAs, power measures alone are not helpful. Imagine a scenario in which two cell assemblies are located in different areas of the brain but connected by a few long-range connections. If the influence of the first NCA on the second is sufficiently strong, an oscillation in the first cell assembly should evoke the second to engage in oscillating activity of the same frequency. Because of the distance between the NCAs, there will be a time lag between the two oscillations. This sort of long-range synchrony cannot be measured by power enhancements of a single electrode. Rather, one would need a measure that is able to mirror the time lag between the activation of several electrodes. Phase-locking analysis as described by Lachaux and colleagues ${ }^{23}$ uses the phase difference between two recorded signals and quantifies whether this phase difference is constant over time. A perfect coupling of the two signals results in a constant phase difference and is operationalized with a phase-locking value of one. Lower values indicate weaker phase synchronization, and the value of zero reflects no phase coupling at all. In the studies described later we used this logic to describe the global synchronization of NCAs in tinnitus subjects and controls using EEG and MEG.

The main generators making up the EEG/MEG-signals most likely originate from cortical rather than from subcortical areas. There are two reasons for this: First, the electric and magnetic fields as recorded in EEG and MEG decline quadratically with increasing distance. Because the subcortical structures are further away from the surface than the cortical layers, they exert a much weaker signal. Second, as outlined earlier, there is need for at least 10,000 uniformly oriented neurons to build an electric field and thus generate a measurable signal. Activity from such regions can often be represented by a so-called equivalent dipole. ${ }^{24}$ This is the case for most cortical structures, in which the apical dendrites of pyramidal neurons are aligned in parallel, pointing perpendicularly to the cortical surface. In subcortical structures, however, neurons are arranged in nuclei with a more complex manner. Hence the generated field is unlikely to be dipolar, which thus limits its impact on externally recordable signals. Nevertheless, this does not mean it is impossible to record deeper sources (see, e.g., some MEG studies claiming to measure subcortical structures $^{25}$ ).

\section{ABNORMAL CORTICAL ACTIVITY IN TINNITUS}

As outlined earlier, one must integrate measures of local and global synchrony to fully describe the normal and abnormal activity in neuronal cell assemblies of the cortex. To understand the abnormal cortical activity in tinnitus, we conducted a series of studies using both measures in the MEG comparing chronic tinnitus patients with normal healthy controls. These results were used to develop an EEGneurofeedback therapy for the treatment of tinnitus.

\section{ABNORMIAL LOCAL SYNCHRONY IN THE RESTING-STATE}

\section{Alpha Reduction and Delta Enhancement}

Seventeen chronic tinnitus patients and 16 healthy controls participated in this MEG study. ${ }^{26}$ All tinnitus patients reported duration of tinnitus lasting at least 1 year (range, 1 to 17 years). The majority of patients who reported a known cause for the tinnitus cited the etiology as noise trauma or sudden hearing loss. In four cases, tinnitus was perceived bilaterally, in nine cases unilaterally to the left ear, and four times unilaterally to the right ear. Tinnitusrelated distress was assessed using the standardized German Questionnaire "Tinnitus Fragebogen," 27 an adaptation of the "Tinnitus Questionnaire."28 According to this questionnaire, 11 patients suffered from slight, 2 patients from moderate, 2 from severe, and 2 from very severe tinnitus distress. There was no significant age difference between the tinnitus and the control group. All participants 
were instructed to lie quietly in a comfortable supine position, to keep their eyes open, and to stay awake without thinking any specific thoughts for 5 minutes. This resting-state activity was recorded using a 148-channel whole-head MEG system (see Weisz et $\mathrm{al}^{26}$ for more technical details).

In a first explorative step, the power spectrum ( 1 to $35 \mathrm{~Hz})$ of all sensors was calculated to compare the tinnitus to the control group.
Figure 1 (middle) depicts the frequency spectra of both groups. In the control subjects, there was a prominent peak in the $\alpha$ ( 8 to $12 \mathrm{~Hz}$ ) frequency range, which was strongly reduced in the tinnitus group. The power in the delta ( 1 to $4 \mathrm{~Hz}$ ) range, in contrast, was enhanced in tinnitus relative to control subjects. Overall, tinnitus is characterized by a local desynchronization in the $\alpha$ and enhanced local synchrony in the delta frequency range.
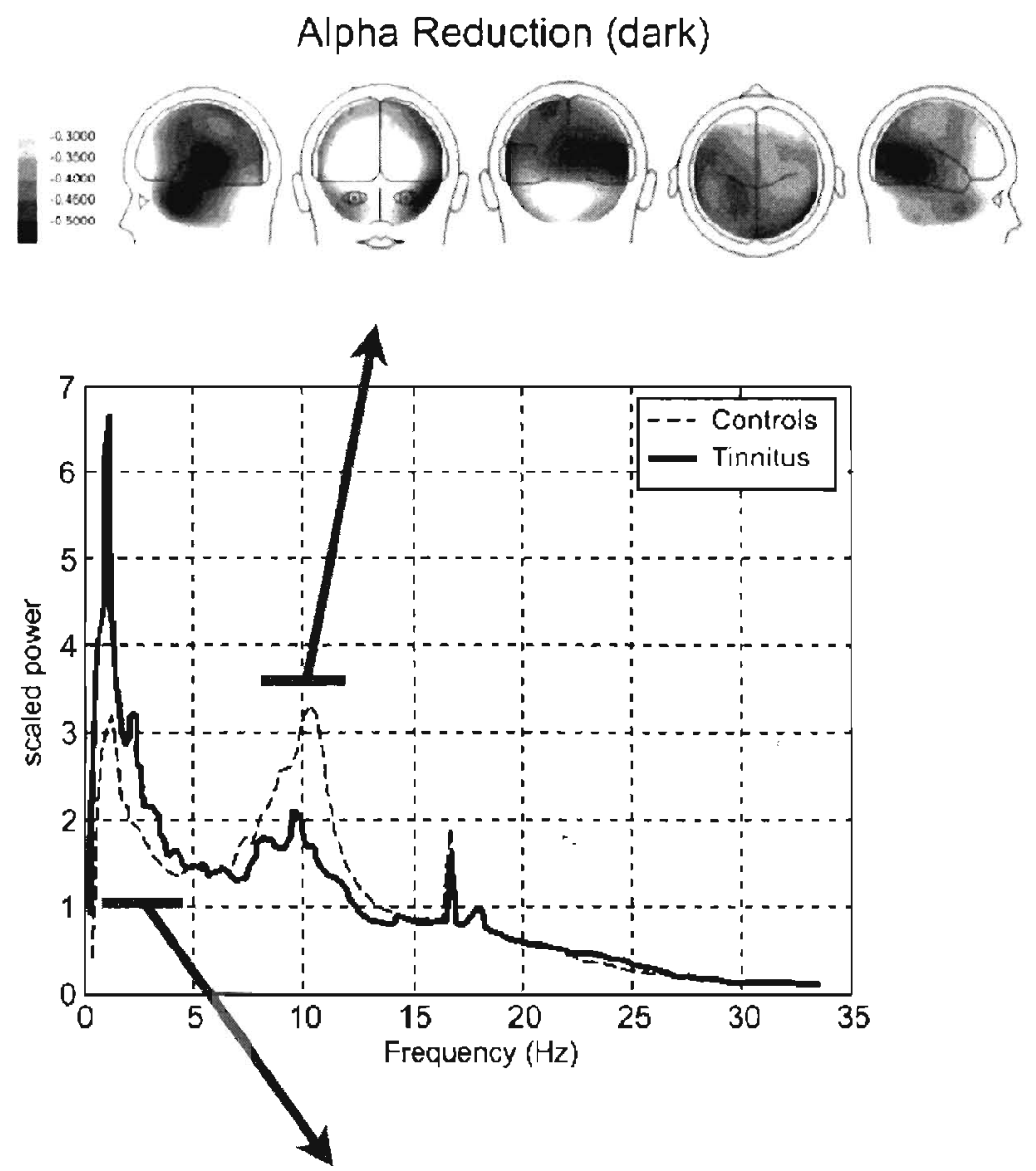

Delta Enhancement (dark)

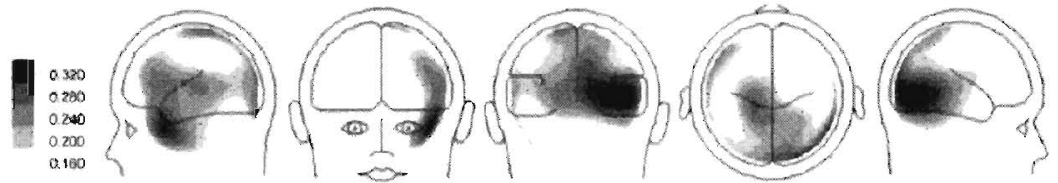

Figure 1 Altered cortical resting-state activity in tinnitus subjects can be characterized by a relative decrease of power in the alpha band and a relative increase of power in the delta frequency band. Middle: Normalized power spectra of the tinnitus and the control group during 5 minutes of resting magnetoencephalography recordings. Top: Difference map showing the spatial distribution of the alpha reduction in tinnitus. Bottom: Difference map depicting the delta enhancement in tinnitus. (Adapted from Weisz et al. ${ }^{26}$ ) 
Because this power spectrum was collected over 148 channels of the whole head, no specific statement about the origin of the abnormal cortical activity can be made. Thus, in a second step, a minimum norm estimation was used to estimate the underlying source activity that generates the signal at the surface sensors. Applying this technique to the data of this study, it was revealed that the $\alpha$ reduction and delta enhancement in tinnitus mostly originate from temporal areas in the cortex (Fig. 1, top and bottom).

Finally, in an attempt to relate this abnormality to the tinnitus symptoms, dipole activity of the alpha and delta frequency bands were correlated with the individual tinnitus distress ratings. To provide a more simplified overview, we only present (Fig. 2) the correlations of the combined frequency index (delta - alpha)/(delta + alpha) with the total score of the Tinnitus Questionnaire. Correlations between the tinnitus distress and the abnormal spontaneous activity of the alpha and delta frequencies were strongest in the right temporal and the left frontal area. Overall, effects were greater for the alpha than for the delta frequency band.

\section{Gamma Enhancement}

The study just described was restricted to frequencies in the range of 1 to $35 \mathrm{~Hz}$. In the second study on local synchrony of NCAs, we included gamma frequencies up to $90 \mathrm{~Hz}^{29} \mathrm{~A}$ sample of 26 tinnitus patients and 21 healthy controls were included in this study. In the tinnitus sample, 7 patients localized their tinnitus predominantly to the right side, 9 to the left side, and 10 subjects reported their tinnitus to be equally loud in both ears. According to the German adaptation of the Tinnitus Questionnaire, ${ }^{27} 16$ patients in this sample suffered from slight tinnitus distress, 4 from moderate, 3 from severe, and 2 from very severe distress.

Again, patients were recorded for 5 minutes with eyes open in the MEG. The data were down-sampled to $450 \mathrm{~Hz}$, and a source montage of eight regional dipoles was generated to concentrate on temporal sources. Two dipoles were located in the left and right auditory cortex, and the six remaining dipoles were placed in the left/right frontal, left/right parietal, and near the anterior and posterior cingulum. The signals from the 148 sensors were projected on this montage with respect to individual head sizes. The purpose of this approach was to restrict the analysis to signals that mainly originate from the auditory cortex. The six additional sources served to capture brain activity occurring in other regions. Thus the locations of these sources were predefined by the investigators and are not a result of a dipole fitting approach. In the following we report only data from temporal areas.

To study the differences in local gamma synchrony between tinnitus and control subjects, we analyzed frequencies in the $40-$ to $90-\mathrm{Hz}$ range originating from auditory areas: The gamma power in the tinnitus group was significantly enhanced $(t(89)=2.9, p<0.01)$ relative to the control group (Fig. 3). There was no significant correlation between gamma band activity and individual distress ratings. To investigate the mutual interrelation of the delta, $\alpha$, and gamma frequency band, the individual values of the three frequency bands are plotted against each other in Fig. 4. The left panel in this figure again demonstrates the findings of our first study: increased delta power accompanies a decline in alpha power. The middle panel shows a strong negative relationship between alpha and gamma band activity. In the right panel the interaction of delta and gamma activity appears to be nonlinear, with extremely low and

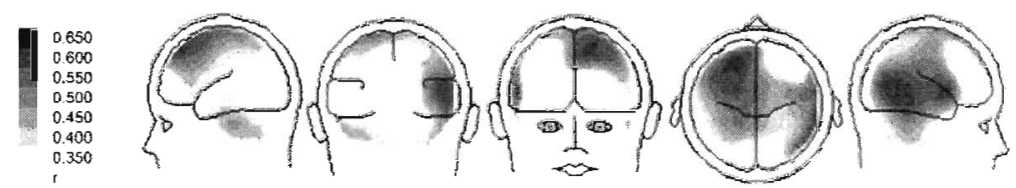

Figure 2 Spatial distribution of dipoles reveals significant correlations of the alpha reduction/delta enhancement with tinnitus distress. Correlations were strongest in the right temporal and the left frontal cortical region. (Adapted from Weisz et al. ${ }^{26}$ ) 


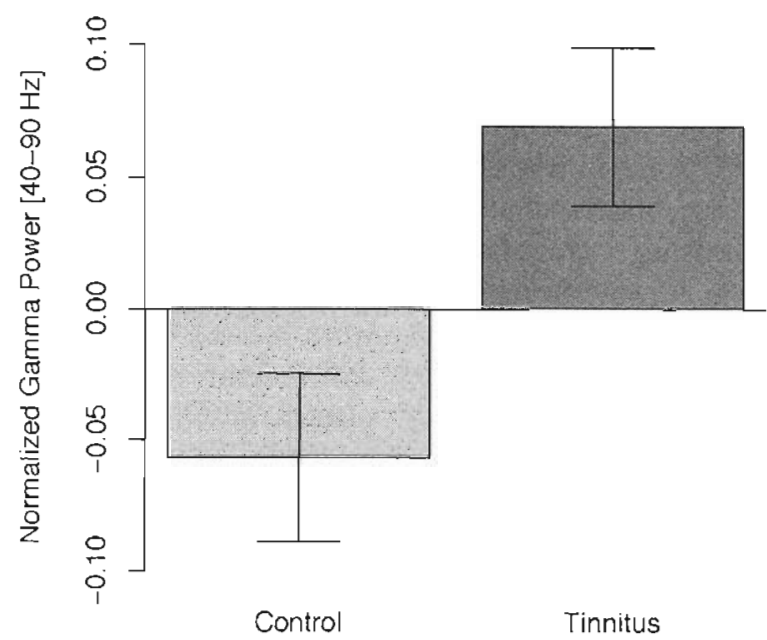

Figure 3 Group difference of the mean gamma power in the 40 - to $90-\mathrm{Hz}$ range recorded during 5 minutes of resting magnetoencephalography with eyes open. Gamma band activity in the tinnitus group was significantly increased relative to the control group.

high delta values associated with lower gamma, whereas midrange delta activity is associated with greater gamma band activity. Later we describe another study on the interaction of slow-wave activity and gamma power in the temporal cortex.

\section{NEUROFEEDBACK WITH TINNITUS: A THERAPEUTIC INTERVENTION FOR RESHAPING ABNORMAL CORTICAL ACTIVITY}

Neurofeedback provides real-time feedback of cortical activity to the subject. The goal of this technique is to provide the subject with the opportunity to manipulate his or her own cortical activity into a desired direction. If the user is successful in this manipulation, positive feedback is provided. Neurofeedback is an operational learning process: There are no instructions to the subjects on how to manipulate their own brain activity; the users gain cognitive control over their cortical activity by contingent feedback about the success or failure of their behavior.

We based our neurofeedback intervention on the findings of abnormal cortical activity in tinnitus as described earlier. Because abnormalities in the alpha and delta range are associated with tinnitus distress, the aim of the treatment was to reshape cortical activity in these frequency bands. We used an electrode setup that was designed to record brain responses mostly originating from the auditory cortex. Online
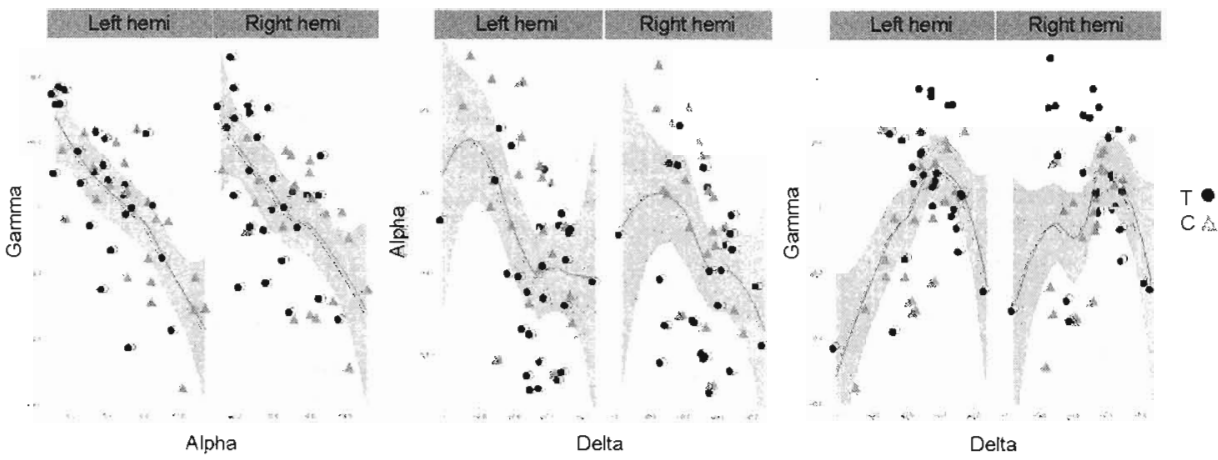

Figure 4 Mutual interaction of delta, alpha, and gamma activity in-temporal areas. Left: alpha-delta. Middle: gamma-alpha. Right: gamma-delta. Dots indicate tinnitus patients; triangles indicate control subjects. Relationships for the left and right temporal cortex are shown. 
feedback of the EEG signal was provided visually in form of a cartoon fish presented on a monitor in front of the patient. The participants were asked to move the feedback signal into a target area reflecting 'normal' cortical activity.

\section{Neurofeedback as a Treatment for Chronic Tinnitus}

In this study of neurofeedback, ${ }^{30}$ we included 21 chronic tinnitus patients ( 9 women) ages 31 to 62 years (mean, 48 years). The mean tinnitus duration in this sample was 8.7 years $(\mathrm{SE} \pm 1.6)$, the average loudness of the tinnitus sound, as matched via a $1-\mathrm{kHz}$ tone, was $25 \mathrm{~dB}$ hearing level ( $\mathrm{SE} \pm 2.6$ ). Overall, the patients in this study reported slight levels of tinnitus distress according to the German version of the Tinnitus Questionnaire: 26.5 points $( \pm 3.4)$. The EEG signals were recorded from four electrodes attached to frontocentral positions (F3, F4, FC1, and FC2). The patients were assigned to one of the following three feedback protocols: (1) reward for increments of the ratio between alpha ( 8 to $12 \mathrm{~Hz}$ ) and delta power ( 3 to $4 \mathrm{~Hz})$; (2) reward for increments of power in the alpha band; (3) reward for reduction of power in the delta band. Ten individual sessions for every participant were conducted that lasted 30 minutes each, distributed across 4 weeks. Before and after the training sessions, we recorded 5 minutes of resting EEG via the four frontocentral electrodes. Except for the different protocols, the design of the study was similar for every participant.

To estimate the specific effects of the neurofeedback training on tinnitus, we compared the results from the neurofeedback training with results from a training approach, called frequency discrimination training (FDT). The FDT also consisted of 10 sessions: however, the treatment time was longer at 120 minutes per session. Twenty-seven patients with chronic tinnitus participated in the FDT. The patients of the FDT group were an average of 53 years of age (ranging from 24 to 65 years) with tinnitus duration of 9.1 years ( $\mathrm{SE} \pm 1.48$, range, 1 to 32 years). Patients were to differentiate between two pure tones with the distance between the tones decreasing with increasing success. The frequency range of the training tones lay in the hearing loss range, thus activating the deprived zones of the tonotopic map with behaviorally relevant input. Furthermore, patients wore a hearing aid that enhanced frequencies in the hearing loss region.

To compare the two groups with respect to the tinnitus distress, a repeated measures anal$y$ sis of variance (ANOVA) was performed with the between-subject factor of "group" (neurofeedback versus frequency discrimination), and the within factor of "time" (pre, post, and 6-month follow-up) revealed a significant group*time interaction $(F(2,72)=3.73, p=$ 0.008 ). There was no significant reduction of tinnitus distress in the FDT sample: hence we concluded that the improvements in the neurofeedback group were a result of successful intervention and not simply a placebo treatment effect.

Patients in the neurofeedback group demonstrated a considerable ability to regulate the amplitudes of their brain waves by means of the neurofeedback training procedure. Overall, the alpha-delta ratio increased significantly across all sessions $(t(20)=-3.34$, $p=0.003$ ) with an average of $71 \%$ (range, -32 to $325 \%$ ). The tinnitus intensity decreased substantially from $25 \mathrm{~dB}(\mathrm{SE} \pm 2.6)$ before to $16.9 \mathrm{~dB}(\mathrm{SE} \pm 2.9)$ after the treatment. Contrasting the pre- and post-measuring points revealed a highly significant reduction of tinnitus intensity $(F(1,13)=16, p=.008)$. The reduction of the perceived tinnitus intensity and the normalization of the alpha-delta ratio are strongly correlated with $r=-0.74 \quad(p<0.001$; see Fig. 5). Thus the patients who successfully modified their oscillatory brain activity were the ones who benefited most from the therapy. Tinnitus-related distress was also noticeably reduced in the neurofeedback sample from a mean of 26.5 points on the Tinnitus Questionnaire $(\mathrm{SE} \pm 3.35)$ at the beginning to 19 points $(\mathrm{SE} \pm 3.3)$ at the end of the last session (Fig. 5B). After 6 weeks the sample showed a very slight increase in the distress score (20 points; SE: \pm 4.14 ) that persisted up to 6 months (20.5; SE: \pm 5.38 ).

Figure $5 \mathrm{~A}$ shows a strong correlation between tinnitus intensity reduction and 

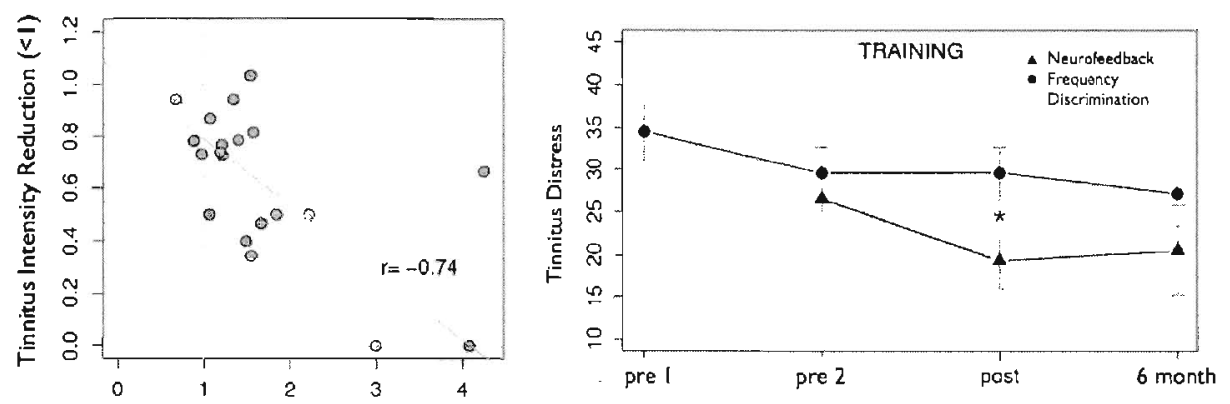

A

A/D Normalization (>1)

B

Figure 5 (A) Normalization of the alpha-delta ratio is negatively associated with the reduction of tinnitus intensity after therapy $(r=-0.74 ; p<0.001)$. Values of the intensity reduction and the alpha/delta enhancement are displayed as the ratio of the measure after the training divided by the respective measure before the training. (B) Mean values ( \pm SE) of tinnitus distress for the frequency discrimination group (circles) and the neurofeedback group (triangles). The gray bar displays the training period. The asterisk indicates a significant difference between the groups after the training period, whereas there is no significant difference at pre 2 and 6 months follow-up between the two training groups. (Adapted from Dohrmann et al. ${ }^{30}$ ) SE, standard error of the mean.

enhancement of the alpha-delta ratio. An enhancement of this ratio, however, does not reflect definitively a simultaneous change of both bands. It could be also a result of readjustment in only one of the two frequency bands. To resolve this issue, patients were classified in four groups based on their ability to modify alpha and delta irrespective of the feedback protocol. This analysis shows that participants who modified both bands simultaneously showed the strongest tinnitus reduction or even abolishment of the tinnitus sensation (Fig. 6). Tinnitus intensity reduction in this group was 78\%. Comparisons with the "delta", "alpha", and "no change" cluster revealed significant group differences for the latter two groups $(\mathrm{t}(7)=1.6, p=0.07 ; \mathrm{t}(9)=2.9$, $p<0.01 ; \mathrm{t}(6)=2.7, p=0.02$; respectively).

Based on our previous findings of a negative linear correlation between alpha and gamma power, we hypothesized a decrease of gamma power in those patients that are able to
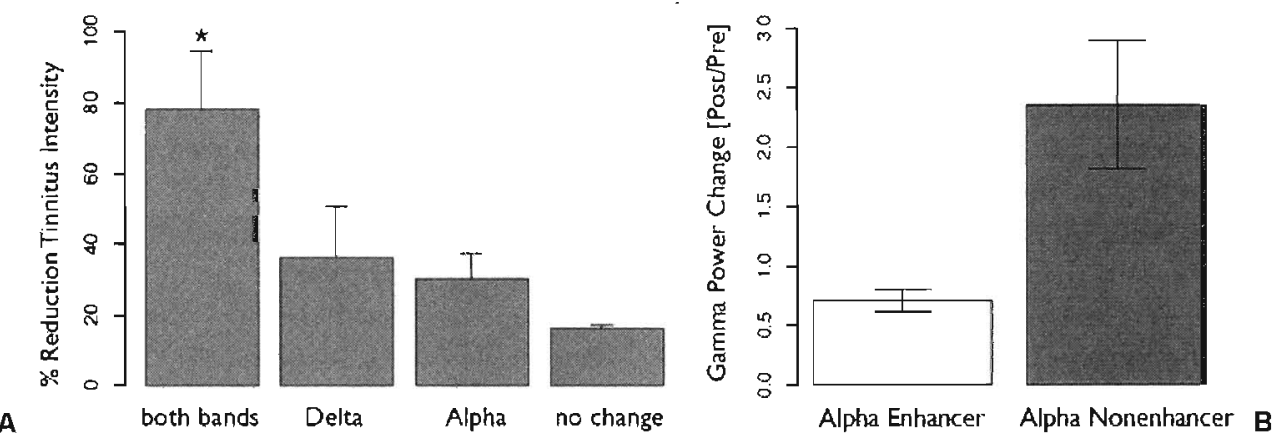

Figure 6 (A) The 21 patients were grouped into four clusters depending on their ability to change their cortical activity in the alpha and delta frequency range. Four subjects were able to alter both frequency bands, six patients only changed their delta activity, seven only the alpha activity, and four participants were not able to modify either of the frequencies. In the "both bands" group, tinnitus intensity was reduced by $78 \%$ (SE \pm 17 ), in the delta group the reduction was $36 \%$ (SE \pm 15 ), in the alpha group the decrease was $30 \%$ (SE \pm 7 ), and in the "no change" group the reduction was only $16 \%$ (SE \pm 1 ). ( B) Change of gamma power that occurred nonintended during the therapy. The group of patients that enhanced their alpha turned to reduce their gamma (mean 0.7; $S E \pm 0.09$ ). The remaining group enhanced gamma power (mean 2.3; $\mathrm{SE} \pm 0.54$ ). (Adapted from Dohrmann et al. ${ }^{30}$ ) $\mathrm{SE}$, standard error of the mean. 
enhance their alpha power. Thus we split the sample in two groups with the first group comprised of patients with a pre-to-post alpha change above the median of the sample and the second group below the median. The change of gamma power in the $30-$ to $45-\mathrm{Hz}$ range was calculated by dividing the post gamma power by the gamma power measured before the therapy. Thus a value of 1 would indicate no change of gamma power as response to the therapy. In the group of "alpha enhancers," the gamma power significantly decreased $(\mathrm{t}(8)=-3.1, p=0.01)$, whereas gamma power increased in the second group $(t(8)=2.4, p=0.04)$. Note there was no feedback given for the gamma power, so the changes in this frequency band occurred concomitantly with the changes in the alpha and/or delta frequency band that the patients intended. Although the gammareduction in the alpha-enhancer group is in line with previous results, the gamma enhancement in the second group alpha surprising. However, this is most likely a consequence of the median split in this case. The group of "alpha nonenhancers" turns out to be heterogenic grouping together of patients who reduced alpha and patients who did not change their alpha at all.

To summarize the results of the first neurofeedback study, we learned (1) the pathological pattern of cortical activity as present in chronic tinnitus patients can be modified via neurofeedback training, (2) those patients able to change activity in the delta and the alpha frequency band simultaneously benefit most from the treatment, and (3) concomitant with the alpha power increase, the gamma power decreased, even though it was not the intention of the treatment protocol.

\section{Abnormal Global Synchrony in the Resting State}

Synchronization between NCAs can occur over long distances in the brain. We wanted to measure global synchrony between cortical areas without prior restrictions by using a brain montage of coarsely defined sources throughout the cortex. We projected signals that were recorded at $148 \mathrm{MEG}$ sensors to sources in the temporal, frontal, and parietal regions of both hemispheres, as well as to the anterior and posterior cingulum. The data were downsampled to $450 \mathrm{~Hz}$, and 1 artifact-free minute was selected for subsequent analyses. A Morlet wavelet $(m-$ factor $=7$ ) was used for estimation of the instantaneous phases in the frequency range of 1 to $90 \mathrm{~Hz}$. This phase information was used to calculate the phase-locking value (PLV), which may be an operationalization for functional neural connectivity. ${ }^{23}$ In this case the phase difference between two signals is calculated and tested for stability across all time points. The PLV (from zero to one) increases the more the distribution within a unit circle deviates from uniformity, with a PLV of one indicating perfect phase coupling between the two signals. An increased PLV between two sources is thus interpreted as an increased synchronization between NCAs of the given brain areas.

In this study, we tested 21 individuals with tinnitus (five women) and 20 normal controls (six women) (W. Schlee and colleagues, unpublished data, 2008). All tinnitus patients suffered from chronic tinnitus with a mean duration of 6.2 years (range, $1-15$ years). Tinnitus severity was assessed with a standardized German questionnaire; ${ }^{27}$ values varied in this sample between slight (4 points) and severe (50 points) distress with an average of 23.6 points.

In the first step of the data analysis, we identified the frequency bands that display a group difference in their resting-state brain network. We normalized the data by dividing each PLV by the mean phase locking over all frequencies and all connections of each single subject. We then averaged the frequency-specific PLVs in both groups. Fig. 7 shows the spectrum of interareal synchronization for both groups: There was a remarkable increase of phase synchronization in the $\alpha$ band ( 9 to $12 \mathrm{~Hz}$ ) for the control group that was completely absent in the tinnitus group. Furthermore, we observed an increase of gamma synchronization in tinnitus that spans a frequency range from 40 to $70 \mathrm{~Hz}$, whereby only the frequency bins in the range from 48 to $54 \mathrm{~Hz}$ remained statistically significant after Bonferroni correction (all $p<0.001$ ). There was no significant group difference in the global synchrony of 


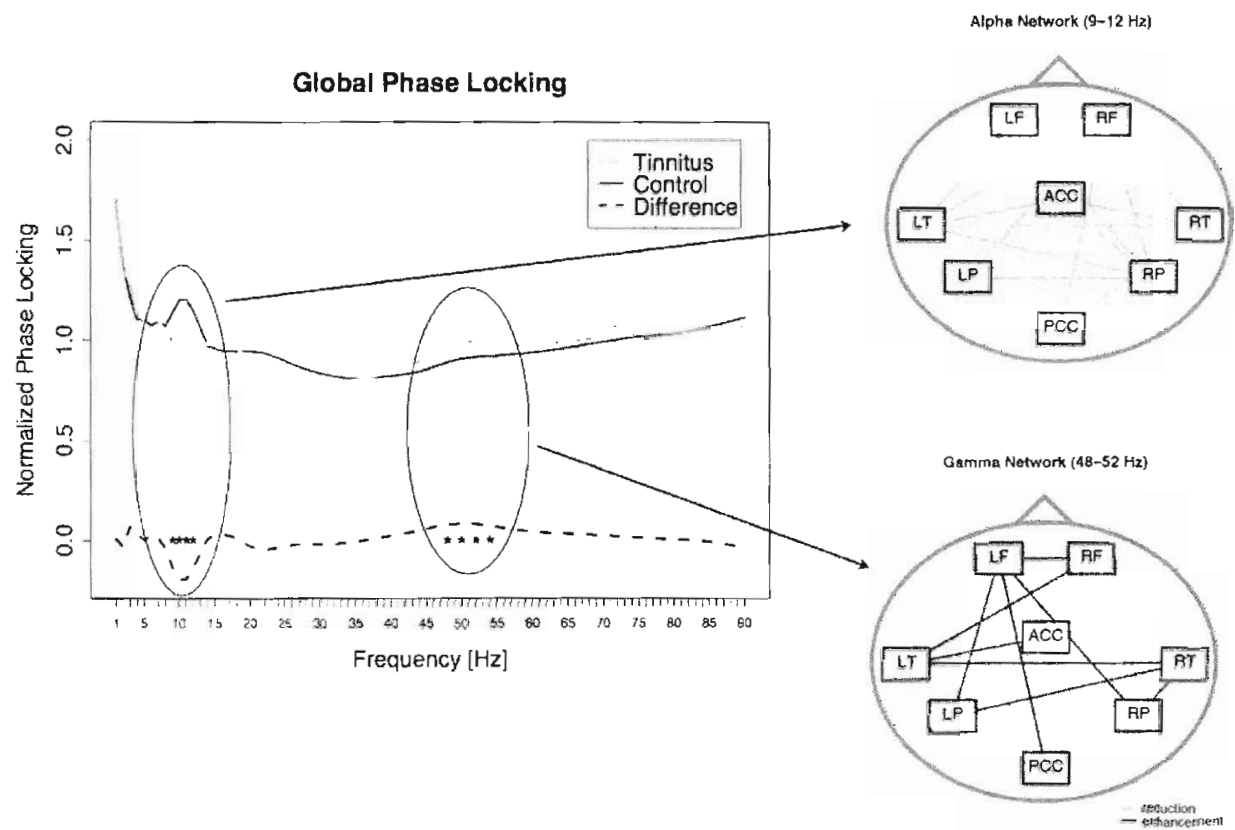

Figure 7 Left panel shows interareal synchronization averaged over all cortico-cortical connections in the resting state. Relatıve to the control group (black line), the tinnitus group (gray line) showed less phase locking in the alpha frequency band and enhancement in the gamma range. Significant deviations from the control group are shown with an asterisk. In the right panel, the magnitude of the group difference for every single connection is indicated by line thickness. (From Schlee et al, unpublished data; 2008.)

the delta frequency range, whereas in studies on the local synchrony of NCAs as described earlier, we found significant enhancement in the delta band.

In a second step, we were interested in the connectivities that account for the group differences in the alpha and gamma bands. The same statistical tests were applied for every single interregional connection, and the result is shown on the right-hand side in Fig. 7. On a group level, the alpha network of relative desynchronization and the gamma network of enhanced synchronization revealed different architectures. Thus the two networks most likely involve different pathways. However, this does not mean they are functionally unrelated.

To test this, we observed the activity within the alpha and gamma networks on a single-subject level. In Fig. 8 we plotted the summed activity of the alpha network against the summed activity of the gamma network, which revealed a strong negative relationship $(\mathrm{r}=-0.71, p<0.001)$ between them. Whenever the synchronization in the alpha network was high in a subject, the synchronization of the gamma network was low. Furthermore, the counterplay of alpha and gamma discriminated well between subjects with and without tinnitus. In tinnitus patients, the alpha synchronies were low, and gamma was high. In control subjects, alpha connectivity dominated, whereas low synchronizations occurred in the gamma range. Calculating a discriminate analysis with cross-validation, we were able to predict group membership based on the network activities with an accuracy of $83 \%$.

\section{INTERACTION OF SLOW-WAVE ACTIVITY AND GAMMA POWER IN THE TEMPORAL CORTEX}

As described earlier, the spontaneous brain activity in the temporal cortex of tinnitus sufferers is characterized by an increase in delta power, a decrease in alpha power, and an increase in gamma power. The enhanced slow-wave activity at a cortical level could be the result of spike bursts from the thalamus, which are conducted to the cortex via thalamocortical afferents. Sensory deprivation marked by enhanced slow-wave activity at the cortical level can lead to an inactivation of intracortical inhibitory 


\section{Individual Network Activations}

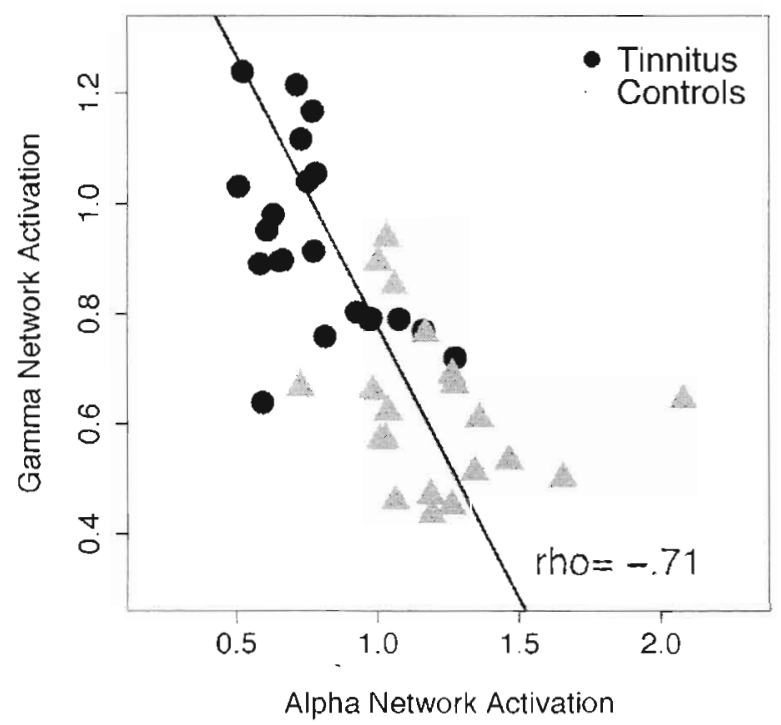

Figure 8 Relationship between the summed activity in the gamma and the alpha network on a singlesubject level. There is a strong negative correlation (Spearman's rank correlation rho $=-0.71 ; p<0.001$ ) between alpha and gamma synchronization. Tinnitus subjects (circles) and control subjects (triangles) are separated well on this spectrum. (From Schlee et al, unpublished data; 2008.)

neurons, which distorts the balanced system of lateral inhibitions in cortical structures. This leads to high-frequency oscillations in a zone between normally afferented and deafferented regions, which is described as the "edge effect." "31,32 According to Jeanmonod and colleagues, ${ }^{33}$ spike bursts in thalamic nuclei occur in regular rhythms of $\sim 4 \mathrm{~Hz}$ and may be a result of hyperpolarization because of deafferentation or overinhibition. These rhythms are associated with positive symptoms in clinical disorders such as neurogenic pain, Parkinson's disease, depression, and tinnitus. $^{31}$ In the tinnitus framework, damage to the hearing system most likely leads to a deafferentation of thalamic neurons with their characteristic frequency in the affected region and thus triggers the cascade of the edge effect described earlier.

To test this hypothesis, we analyzed the spontaneous resting MEG of 26 chronic tinnitus patients ( 6 women; mean age: 45 years. age range. 20 to 65 years) and 21 control subjects with normal hearing (16 women; mean age. 35 years; age range. 23 to 78 years). ${ }^{34}$ The average tinnitus distress in this sample was $27.5(\mathrm{SE} \pm 3.5)$ points on the Tinnitus
Questionnaire, and the overall tinnitus duration was 6.8 years (SE: \pm 1.0$)$. Nine subjects reported tinnitus lateralized to the left ear, seven subjects lateralization to the right ear, and ten patients perceived their tinnitus equally on both sides.

Data were recorded using a 148 wholehead MEG system. Only the signals originating in temporal sources were used for the analysis (see Weisz et $\mathrm{al}^{34}$ for more technical details). First, we selected peaks of increased slow-wave activity in the data stream by applying a Hilbert transformation to the 2 - to $7-\mathrm{Hz}$ bandpass filtered signals. The Hilbert transformation produces a time series of complex numbers, and we calculated the squared modulus of this series for peak selection. We selected those peaks with a magnitude above the median of all points but only when there were no other peaks in the previous 750 milliseconds time window. Approximately 30 peaks fulfilled the criterion for each subject and hemisphere. A trigger was set to these points and the time windows 750 milliseconds pre and 1250 milliseconds post the slow-wave peak of the original data were analyzed with a Morlet wavelet $(\mathrm{m}$-factor $=7$ ). 


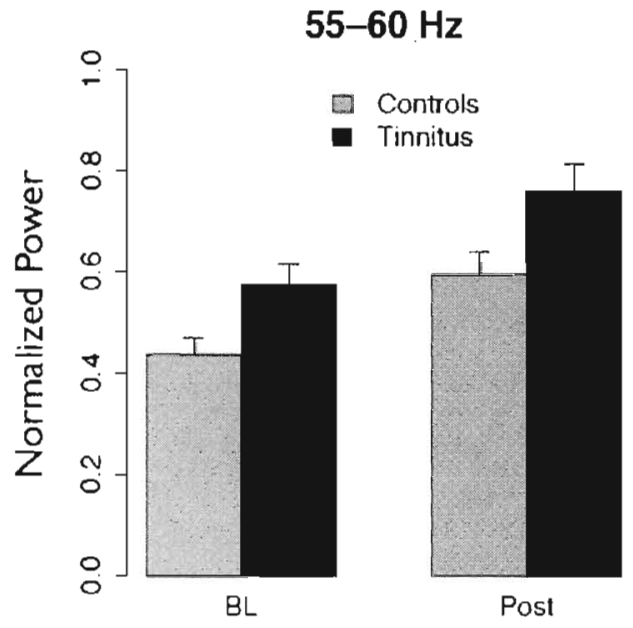

A

Figure 9 (A) Normalized power spectrum ( $\pm \mathrm{SE}$ ) over temporal regions at the baseline (BL) before the slow-wave peak and after it (Post). In both groups, gamma 55- to 60- $\mathrm{Hz}$ power increases as response to the slow-wave activity. At all time points, there is a gamma increase in the tinnitus sample compared with the control group. (B) The laterality index $(\mathrm{LH}-\mathrm{RH}) /(\mathrm{LH}+\mathrm{RH}))$ reflects asymmetrical distribution of gamma power over left and right temporal areas. A positive value indicates lateralization to the left hemisphere. Gamma laterality was absent in subjects with bilateral tinnitus. However, in subjects who perceive unilateral tinnitus, gamma power was lateralized to the contralateral hemisphere. (Redrawn from Weisz et al. ${ }^{34}$ ) SE, standard error of the mean.

Thus we were able to analyze the modulation of gamma power in a time window around a slow-wave peak. Indeed, we found a systematic increase of gamma activity in the $55-$ to $60-\mathrm{Hz}$ frequency range following the low-frequency peak (Fig. 9). The gamma power increased from pre to post by $\sim 33 \%$. This time course appeared in tinnitus patients as well as in control subjects, suggesting that the same neural mechanism occurs in both groups. However, in the tinnitus sample, there was generally more gamma power before and after the slow-wave peaks than in the control group. The permanent enhancement of gamma in the tinnitus group was $\sim 30 \%$. An ANOVA analysis revealed a main effect for time $(\mathrm{F}(1,45)=237.2, p<0.001)$ and a main effect for group $(\mathrm{F}(1,45)=5.4, p=0.03)$; the interaction effect was not significant. To analyze the gamma increase in the tinnitus sample in more detail, we compared the magnitude of gamma power in the left and right temporal cortex. Therefore, a laterality index (LH - RH [left hemisphere-right hemisphere $] /(\mathrm{LH}+\mathrm{RH})$ was calculated to quantify the hemispheric asymmetry in the $55-$ to $60-\mathrm{Hz}$ range. We found a significant linear relationship (linear contrast: $\mathrm{t}=2.9, p<0.008$ ) between the individually perceived location of the phantom sound and the gamma laterality in temporal regions. In the case of unilateral tinnitus, the gamma power was increased contralateral to the reported side. For bilateral tinnitus, there was no lateralization of gamma activity (Fig. 9).

In sum, we found evidence supporting the hypothesis that low-frequency activity, which most likely stems from deafferented regions at the thalamic level, triggers high-frequency responses in the temporal cortex. Highfrequency responses in the gamma range are overall augmented in tinnitus sufferers and are 
Table 1 Summary of Abnormal NCA Activity in Tinnitus

\begin{tabular}{lll}
\hline $\begin{array}{l}\text { Frequency } \\
\text { Band }\end{array}$ & $\begin{array}{l}\text { Local Synchrony } \\
\text { (Temporal Cortex) }\end{array}$ & $\begin{array}{l}\text { Global } \\
\text { Synchrony }\end{array}$ \\
\cline { 2 - 3 } Delta & $\uparrow$ & n.s. \\
Alpha & $\downarrow$ & $\downarrow$ \\
Gamma & $\uparrow$ & $\uparrow$ \\
\hline
\end{tabular}

$\uparrow$, enhancement; $\downarrow$, reduction; n.s.: no significant change.

NCA, neural cell assembly

Results compiled from Weisz $N$, Moratti S, Meinzer $M$, Dohrmann K, Elbert T. Tinnitus perception and distress is related to abnormal spontaneous brain activity as measured by magnetoencephalography. PLOS Med 2005;2:e153; Müller S. Analyse des neuromagnetischen Spektrums bei Tinnitus [thesis|. Konstanz, Germany: University of Konstanz; 2007; Dohrmann K, Weisz N, Schlee $W$, Hartmann Weisz N, Müller $S$, Schlee W. Dohrmann K, Hartmann T, Elbert T. Neurofeedback for treating tinnitus. Prog Brain Res 2007;166:473-554; and Weisz N, Müller S, Schlee W, Dohrmann K, Hartmann T, Elbert $\mathrm{T}$. The neural code of auditory phantom perception. J Neurosci 2007;27:1479-1484.

significantly associated with the perceived tinnitus laterality.

\section{CONCLUSION}

The goal of this research was to investigate pathological changes in the activity of neuronal cell assemblies in tinnitus patients and how these abnormalities relate to the tinnitus percept and the tinnitus distress. We studied the NCA activation on a local and a global level, that is, the synchronization of neural activity within one cortical region and between different cortical regions.

The studies on the local synchrony were mainly focused on temporal regions: We report an enhancement of delta and gamma power as well as a reduction of alpha power compared with healthy control subjects. ${ }^{26,29}$ This deviation correlates with tinnitus distress. ${ }^{26}$ Moreover, neurofeedback therapy leads to normalization of this deviant pattern and a reduction of tinnitus distress and tinnitus loudness. ${ }^{30}$ Furthermore, there is a linear negative relationship between alpha and gamma power; ${ }^{29}$ neurofeedback intervention leads to an enhancement of alpha power and a concomitant reduction of gamma power. ${ }^{30}$ Furthermore, the distribution of gamma power over the left and right temporal cortex accounts for the laterality of the perceived tinnitus. ${ }^{34}$
Studying the synchronous activity of NCAs between cortical regions in tinnitus reveals a reduction of long-range synchrony in the alpha range and an enhancement of long-range synchrony in the gamma range relative to normal controls. The global alpha network activity is negatively associated with the gamma network activity, and this pattern discriminates well between the tinnitus and the control group (W. Schlee and colleagues, unpublished data, 2008).

The alpha reduction and the gamma enhancement was observed in both studies on the local synchronization of NCA structures and in studies on the global synchronization. The enhancement of slow-wave amplitudes in the delta range, however, was observed only at the local level; enhanced synchrony between cortical regions was not found. Thus the tinnitus-related neuronal cell assemblies that fire in the $\alpha$ and gamma frequency range mainly involve the auditory cortex and also span over distant cortical regions in the brain. On the other side, abnormal NCA activity in delta range is restricted to temporal regions.

In the following we want to propose a model explaining the results:

1. A reduction of afferent input to the thalamus leads to hyperpolarization and makes the thalamic cells switch into the burstfiring mode. ${ }^{35}$ These bursts occur in a slow-wave rhythm of $\sim 4 \mathrm{~Hz}$ and ${ }^{33}$ are relayed to the responding cortex area through thalamocortical conductance. Because tinnitus is mostly associated with profound hearing loss, this leads to a deafferentation and thus hyperpolarization of the medial geniculate bodies (MGBs) at the thalamic level, which results in burst firing of these cells. Most of the MGBfibers project to the temporal region, ${ }^{6}$ so the bursts appear at the cortical level and are detected as enhanced delta power in the corresponding neurons of the auditory cortex.

Thus there are two "groups" of neurons in the auditory cortex. Neurons with their characteristic frequencies in the hearing loss region 
are subject to burst firing by the thalamus. However, neurons with their characteristic frequency corresponding to those of undamaged cochlear regions receive normal input. According to the edge effect as proposed by Llinás, ${ }^{32}$ there will be an increase of gamma activity at the edge between the deafferented and the normally afferented regions of the auditory representational cortex.

That gamma activity frequently comes along with slow-wave activity finds support in the study in which we observed the modulation of gamma oscillations as response of slow-wave peaks. $^{34}$

2. Based on the present finding, however, we assume the relationship is not a direct one but is mediated via a downregulation of inhibitory mechanisms. Adding to the edge-effect model, we hypothesize that these ongoing inhibitory mechanisms or the effects of these mechanisms are expressed as oscillatory activity $\sim 10 \mathrm{~Hz} .^{36}$ All of the studies described in this article are recordings during the resting state (i.e., there is no specific stimulation or task given to the subjects). In the normal and healthy brain, this resting state is characterized by a prominent synchronization of NCAs in the alpha range (Figs. 1 and 7). Synchronized $\alpha$ activity is assumed to be an indicator for active inhibition of cortical activity, whereas alpha desynchronization reflects a release of inhibition. ${ }^{36,37}$ Support for this view comes from studies that show event-related $\alpha$ desynchronization in brain regions that are relevant for a cognitive task together with an event-related alpha synchronization in brain regions that are irrelevant for the task. ${ }^{38-40}$ We assume that the same mechanisms account for the resting state and here explain the gamma synchronization in tinnitus. For the auditory cortex this model has been recently elaborated by Weisz et $\mathrm{al}^{36}$ (now termed "synchronization by loss of inhibition modulation," SLIM), which assigns an active inhibitory functional role of $\alpha$ even under rest. The SLIM model predicts the emergence of spontaneous synchronization of neuronal activity at points along the tonotopic map in which inhibitory loss is strong enough and at the same time deprivation-related hyperpolarization not too pronounced. Local synchronization expressed as high-frequency (gamma) oscillation is assumed to be a necessary condition for conscious awareness. ${ }^{41}$ On a perceptual level, the SLIM model accounts for the fact that even though hearing losses in tinnitus are frequently very broadband, the percept is usually described as tonal or at least a narrow band noise with a pronounced pitch. However, local synchronization is not a sufficient condition for conscious perception but needs the coordinated action of low-level sensory brain regions and higher-order areas. Extending the original SLIM model we assume that in addition to regulating synchronization at a local level, alpha desynchronization generally gives gamma oscillations the opportunity to synchronize over long distances. This is elaborated in Fig. 10 .

3. Not all sounds nor all pictures or body sensations that are processed in the sensory system also reach the level of conscious awareness. It has been proposed that conscious and unconscious perception depends on the extension of synchronization in neuronal assemblies. ${ }^{42}$ According to this assumption, conscious perception would require long-distance synchronization of neuronal activity; however, in the case of unconscious perception there would be a short-range synchronization of neural activity. In fact, several studies support this view by showing that global synchronization in the beta or gamma range is correlated with conscious perception. ${ }^{43,44}$ Therefore we assume that global gamma synchronization is needed to get a conscious perception of the tinnitus sound.

Thus we propose that the conscious perception of tinnitus is a consequence of two factors: First, a deafferentation of thalamic (MGB) cells leads to burst firing of the thalamus and gamma activity in the temporal cortex mediated via a circumscribed knockout of inhibitory processes reflected in a reduced alpha level. Second, a global desynchronization of alpha oscillations 


\section{high alpha}

\section{NCA firing activity at site 1 \\ NCA firing activity at site 2 \\ synchronized activity of NCA 1 and 2}

low alpha

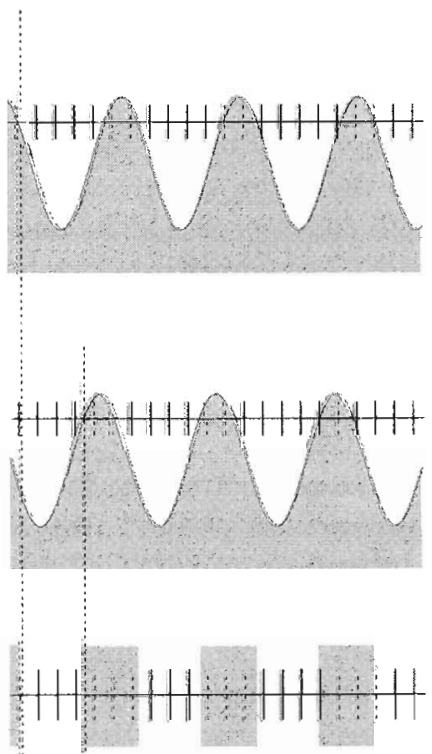

Figure 10 Illustration of the interaction between alpha and gamma synchronization. Columns 1 and 2 show two conditions of high and low alpha power, respectively. Lines 1 and 2 give the gamma firing activity of two NCAs that are located at different sites in the brain (for illustration purposes, the time lag between the two sites was set to 0 milliseconds). The rhythmic changes of alpha result in inhibition of the gamma cells (dotted vertical segments) or firing due to release of inhibition (solid vertical segments). Gamma synchrony between site 1 and 2 occurs only if inhibition is released at both sites simultaneously (illustrated in the bottom line). Thus a global decrease of alpha power as shown in the right column results in enhanced intersite synchronicity. According to this model, an increase of alpha power at either of the two sites would be sufficient to disturb long-range gamma synchronization. Thus the brain can selectively prevent parts of the brain from being incorporated in such a cortical network. (Adapted from Klimesch et al. ${ }^{37}$ )

favors the emergence of long-range gamma synchronization and thus leads to the conscious perception of the tinnitus.

Within this framework are several opportunities for therapeutic intervention. Here we discuss only a few of them. An interruption of the synchronized gamma network should stop the conscious perception of the tinnitus sound. We suppose that transcranial magnetic stimulation and cortical stimulation of the auditory cortex via implanted electrodes ${ }^{45-47}$ could work that way. The problem of this intervention, however, is the architecture of the network. As stated earlier, most brain networks are constructed like small-world networks, which are very tolerant to random attacks. Eliminating one node does not destroy the whole network and is compensated by other nodes. To interrupt the network reliably, we rather need to destroy one or more hubs in the network. Stimulation at several sites of this network at the same time could be a way to overcome this problem. Scientifically, imaging such putative hubs poses a fascinating challenge that greatly exceeds the conventional approaches of analyzing oscillatory brain responses. Deeper insight into this issue will be also therapeutically rewarding as potential targets of brain stimulation.

Another possible intervention is to increase synchronization within the global alpha network. This would increase inhibition and hence hinder global gamma synchronization. So far, we do not know the origin of the alpha desynchronization in tinnitus. However, it has been suggested that alpha activity can be influenced deliberately by top-down processes of the subject. ${ }^{30,36,37,48}$ The neurofeedback approach described here could be one way to modulate the alpha network. In this study, we used the 
temporal cortex as the target for alpha enhancement. Because the temporal cortex is an important hub in the tinnitus-related alpha network, this seems to be a good target for the intervention. One way to improve this method further could be to invent a technique that targets the entire alpha network. Also a combined feedback of the alpha and gamma network activity could help patients learn control over the tinnitus.

\section{ACKNOWLEDGMIENTS}

This work was supported by the Deutsche Forschungsgemeinschaft and the Tinnitus Research Initiative.

We want to thank Fernanda Fernandes, Simona Müller, Ursula Lommen, Christiane Wolf, and Barbara Awiszus for their help on the data acquisition; Patrick Berg and Christian Wienbruch for advice on the data analysis; Christina Robert for help with this manuscript; and Valeska Benz for help designing the figures.

\section{ABBREVIATIONS}

$\begin{array}{ll}\text { ACC } & \text { anterior cingulate cortex } \\ \text { ANOVA } & \text { analysis of variance } \\ \text { EEG } & \text { electroencephalography } \\ \text { FDT } & \text { frequency discrimination training } \\ \text { LH } & \text { left hemisphere } \\ \text { LT } & \text { left temporal cortex } \\ \text { MEG } & \text { magnetoencephalography } \\ \text { MGB } & \text { medial geniculate bodies } \\ \text { NCA } & \text { neuronal cell assemblies } \\ \text { PLV } & \text { phase-locking value } \\ \text { RH } & \text { right hemisphere } \\ \text { RT } & \text { right temporal cortex } \\ \text { SE } & \text { standard error of the mean } \\ \text { SLIM } & \text { synchronization by loss of inhib- } \\ & \text { ition model }\end{array}$

\section{REFERENCES}

1. Heller AJ. Classification and epidemiology of tinnitus. Otolaryngol Clin North Am 2003;36: $239-248$
2. Dobie RA. Depression and tinnitus. Otolaryngol Clin North Am 2003;36:383-388

3. Dandy WE. The surgical treatment of intracranial aneurysms of the internal carotid artery. Ann Surg 1941;114:336-340

4. Silverstein H. Transmeatal labyrinthectomy with and without cochleovestibular neurectomy. Laryngoscope 1976;86:1777-1791

5. Berliner KI, Shelton C, Hitselberger WE, Luxford WM. Acoustic tumors: effect of surgical removal on tinnitus. Am J Otol 1992;13:13-17

6. Eggermont JJ, Roberts LE. The neuroscience of tinnitus. Trends Neurosci 2004;27:676-682

7. Kaltenbach JA. The dorsal cochlear nucleus as a participant in the auditory, attentional and emotional components of tinnitus. Hear Res 2006; 216-217:224-234

8. Mirz F, Brahe Pedersen C, Ishizu K, Johannsen P, et al. Positron emission tomography of cortical centers of tinnitus. Hear Res 1999;134:133144

9. Mühlau M, Rauschecker JP, Oestreicher E, et al. Structural brain changes in tinnitus. Cereb Cortex 2006;16:1283-1288

10. Lee YJ, Bae SJ, Lee SH, et al. Evaluation of white matter structures in patients with tinnitus using diffusion tensor imaging. J Clin Neurosci 2007;14: 515-519

11. Mühlnickel $W$, Elbert $T$, Taub $E$, Flor $H$. Reorganization of auditory cortex in tinnitus. Proc Natl Acad Sci U S A 1998;95:10340-10343

12. Noreña AJ, Eggermont JJ. Changes in spontaneous neural activity immediately after an acoustic trauma: implications for neural correlates of tinnitus. Hear Res 2003;183:137-153

13. König $O$, Schaette $R$, Kempter $R$, Gross $M$. Course of hearing loss and occurrence of tinnitus. Hear Res 2006;221:59-64

14. Braitenberg V, Schutz A. Cortex: Statistics and Geometry of Neuronal Connectivity. 2nd ed. Berlin, Germany: Springer-Verlag; 1998

15. Hebb DO. The Organization of Behavior. A Neuropsychological Theory. New York, NY: J. Wiley \& Sons; 1949

16. Ioannides AA. Dynamic functional connectivity. Curr Opin Neurobiol 2007;17:161-170

17. Laughlin SB, Sejnowski TJ. Communication in neuronal networks. Science 2003;301:1870-1874

18. Watts DJ, Strogatz SH. Collective dynamics of 'small-world' networks. Nature 1998;393:440-442

19. Bassett DS, Meyer-Lindenberg A, Achard S, Duke T, Bullmore E. Adaptive reconfiguration of fractal small-world human brain functional networks. Proc Natl Acad Sci U S A 2006;103: 19518-19523

20. Micheloyannis S, Pachou E, Stam CJ, et al. Small-world networks and disturbed functional 
connectivity in schizophrenia. Schizophr Res 2006; 87:60-66

21. Stam CJ. Functional connectivity patterns of human magnetoencephalographic recordings: a 'smallworld' network? Neurosci Lett 2004;355: 25-28

22. Berger H. Über das Elektrenkephalogramm des Menschen. Arch f Psychiatr 1929;87:527-570

23. Lachaux JP, Rodriguez E, Martinerie J, Varela FJ. Measuring phase synchrony in brain signals. Hum Brain Mapp 1999;8:194-208

24. Elbert T. Neuromagnetism. In: Andrä W, Nowak $\mathrm{H}$, eds. Magnetism in medicine. London, United Kingdom: J. Wiley \& Sons; 1998:190-262

25. Jerbi K, Lachaux JP, N'Diaye K, et al. Coherent neural representation of hand speed in humans revealed by $\mathrm{MEG}$ imaging. Proc Natl Acad Sci U S A 2007;104:7676-7681

26. Weisz N, Moratti S, Meinzer M, Dohrmann K, Elbert $T$. Tinnitus perception and distress is related to abnormal spontaneous brain activity.as measured by magnetoencephalography. PLoS Med 2005;2:e153

27. Goebel G: Hiller W. Tinnitùs Fragebogen (TF): Ein Instrument zur Erfassung von Belastung und Schweregrad bei Tinnitus. Göttingen, Germany: Hogrefe; 1998

28. Hallam R. Psychological approaches to the evaluation and management of tinnitus distress. In: Hazell J, ed. Tinnitus. London, United Kingdom: Churchill and Livingston; 1986:1-50

29. Müller S. Analyse des neuromagnetischen Spektrums bei Tinnitus [diploma thesis]. Konstanz, Germany: University of Konstanz; 2007

30. Dohrmann K, Weisz N, Schlee W, Hartmann T, Elbert T. Neurofeedback for treating tinnitus. Prog Brain Res 2007;166:473-554

31. Llinás RR, Ribary U, Jeanmonod D, Kronberg E, Mitra PP. Thalamocortical dysrhythmia: a neurological and neuropsychiatric syndrome characterized by magnetoencephalography. Proc Natl Acad Sci U S A 1999;96:15222-15227

32. Llinás RR, Steriade M. Bursting of thalamic neurons and states of vigilance. J Neurophysiol 2006;95:3297-3308

33. Jeanmonod D, Magnin M, Morel A. Lowthreshold calcium spike bursts in the human thalamus. Common physiopathology for sensory, motor and limbic positive symptoms. Brain 1996; 119:363-375

34. Weisz N, Müller S, Schlee W, Dohrmann K, Hartmann T, Elbert T. The neural code of auditory phantom perception. J Neurosci 2007;27:14791484

35. Sherman SM. Tonic and burst firing: dual modes of thalamocortical relay. Trends Neurosci 2001; $24: 122-126$
36. Weisz N, Dohrmann K, Elbert T. The relevance of spontaneous activity for the coding of the tinnitus sensation. Prog Brain Res 2007;166: $61-70$

37. Klimesch W, Sauseng P, Hanslmayr S. EEG alpha oscillations: the inhibition-timing hypothesis. Brain Res Rev 2007;53:63-88

38. Pfurtscheller G, Klimesch $W$. Event-related desynchronization during motor behavior and visual information processing. Electroencephalogr Clin Neurophysiol Suppl 1991;42:58-65

39. Sauseng P, Klimesch W, Stadler W, et al. A shift of visual spatial attention is selectively associated with human EEG alpha activity. Eur J Neurosci 2005;22:2917-2926

40. Worden MS, Foxe JJ, Wang N, Simpson GV. Anticipatory biasing of visuospatial attention indexed by retinotopically specific alpha-band electroencephalography increases over occipital cortex. J Neurosci 2000;20:RC63

41. Gross J, Schnitzler A, Timmermann L, Ploner M. Gamma oscillations in human primary somatosensory cortex reflect pain perception. PLoS Biol 2007;5:e133

42. Dehaene S, Changeux JP, Naccache L, Sackur J, Sergent C. Conscious, preconscious, and subliminal processing: a testable taxonomy. Trends Cogn Sci 2006;10:204-211

43. Gross J, Schmitz F, Schnitzler I, et al. Modulation of long-range neural synchrony reflects temporal limitations of visual attention in humans. Proc Natl Acad Sci USA 2004;101: 13050-13055

44. Melloni L, Molina C, Pena M, Torres D, Singer W, Rodriguez E. Synchronization of neural activity across cortical areas correlates with conscious perception. J Neurosci 2007;27: 2858-2865

45. De Ridder D, De Mulder G, Verstraeten E, et al. Primary and secondary auditory cortex stimulation for intractable tinnitus. ORL J Otorhinolaryngol Relat Spec 2006;68:48-54

46. Langguth $B$, Kleinjung $T$, Marienhagen $J$, et al. Transcranial magnetic stimulation for the treatment of tinnitus: effects on cortical excitability. BMC Neurosci 2007;8:45

47. Londero A, Langguth B, De Ridder D, Bonfils P, Lefaucheur JP. Repetitive transcranial magnetic stimulation (rTMS): a new therapeutic approach in subjective tinnitus? Neurophysiol Clin 2006; 36:145-155

48. Thut G, Nietzel A, Brandt SA, Pascual-Leone A. Alpha-band electroencephalographic activity over occipital cortex indexes visuospatial attention bias and predicts visual target detection. J Neurosci 2006;26:9494-9502 